\title{
The Effects of Communication and Visual Range on Multi-Robot Repeated Boundary Coverage
}

\author{
Pooyan Fazli \\ Department of Computer Science \\ University of British Columbia \\ Vancouver, BC, Canada \\ pooyanf@cs.ubc.ca
}

\author{
Alan K. Mackworth \\ Department of Computer Science \\ University of British Columbia \\ Vancouver, BC, Canada \\ mack@cs.ubc.ca
}

\begin{abstract}
We address the problem of repeated coverage by a team of robots of the boundaries of a target area and the structures inside it. The robots have limited visual and communication range. Events may occur on any parts of the boundaries and may have different importance weights. In addition, the boundaries of the area and the structures are heterogeneous, so that events may appear with varying probabilities on different parts of the boundary, and these probabilities may change over time. The goal is to maximize the reward by detecting the maximum number of events, weighted by their importance, in minimum time. The reward a robot receives for detecting an event depends on how early the event is detected. To this end, each robot autonomously and continuously learns the pattern of event occurrence on the boundaries over time, capturing the uncertainties in the target area. Based on the policy being learned to maximize the reward, each robot then plans in a decentralized manner to select the best path at that time in the target area to visit the most promising parts of the boundary. The performance of the learning algorithm is compared with a heuristic algorithm for the Travelling Salesman Problem, on the basis of the total reward collected by the team during a finite repeated boundary coverage mission. Moreover, the effects of robots' visual range and communication among the robots on the performance of the proposed algorithms are also investigated.
\end{abstract}

Keywords: Multi-Robot Boundary Coverage, Uninformed Boundary Coverage, Informed Boundary Coverage, Teamwork, Coordination

\section{INTRODUCTION}

Multi-Robot Boundary Coverage is a challenging problem with various applications including surveillance and monitoring, cleaning, intrusion detection and facility inspection. In this task, a team of robots cooperatively visits (observes or sweeps) the boundaries of the target area and the structures inside it. The goal is to build efficient paths for all the robots which jointly ensure that each point on the boundaries is visited by at least one of the robots. The Boundary Coverage [1] is a variant of the Area Coverage [2]-[4] problem, in that the aim is to cover just the boundaries, not the entire area.

There are two classes of Boundary Coverage problems:

- Single Coverage: The aim is to cover the boundary until all its accessible points of interest have been visited at least once, while minimizing the time, sum/maximum length of the paths/tours generated for the robots, or balancing the workload distribution among the robots [5].

- Repeated Coverage: The goal is to cover all the accessible points of interest on the boundary repeatedly over time, while maximizing the frequency of visiting points, minimizing the weighted average event detection time, or detecting the maximum number of events/intruders. Visiting the points on the boundary can be accomplished with uniform or non-uniform frequency, depending on the priorities of different parts of the boundary [6], [7].

In this paper, we address the Multi-Robot Repeated Boundary Coverage problem with the following specifications:

- The environment is a simple polygon consisting of rectilinear or non-rectilinear polygonal structures.

- The 2D map of the environment is given a priori.

- An arbitrary number of robots is involved in the coverage mission.

- The robots are equipped with a panoramic visual sensor with limited range.

- The robots have limited communication range.

- The events may occur on any part of the boundary.

- The events may have different types. Each event type has its own importance weight.

- The boundary is heterogeneous, in that events of one type may occur with varying probabilities on different parts of the boundary, and this probability may change over time.

- A robot can detect an event if the event is within the visual range of the robot.

- Once a robot detects an event, the event is discarded from the boundary.

- A robot is aware of the types of the events and their importance weights once it detects the events.

- The robots are not a priori aware of the probability distribution of the events occurrence on the boundaries.

- The reward a robot receives for detecting an event depends on how soon the event is detected. At each time step after the event occurrence, the detection reward of the event is decreased by a multiplicative discount factor.

Definition 1. Event Type: $m$ types of events may occur on the boundary. The set of all events types is $E=\left\{E_{1}, E_{2}, \ldots, E_{m}\right\}$. Similarly, an event of type $E_{i}$ is denoted as $e_{i}$.

Definition 2. Event Importance: The importance degree of an event of type $E$ is given by weight $(E)$. It is assumed that weight $(E) \in(0,1]$ such that 1 is the highest degree of importance. The importance can also be referred to as the priority, in that an event of higher importance should have higher priority of being detected.

To address the problem, two classes of algorithms are pro- 


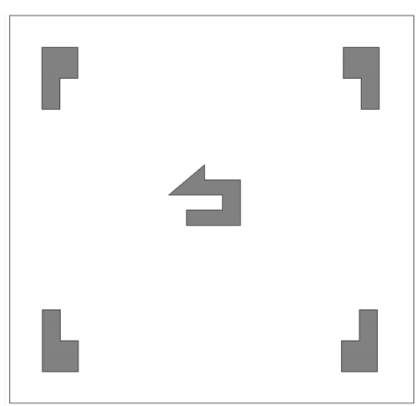

(a) Original Map

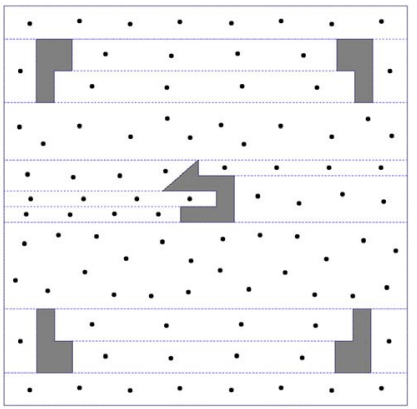

(b) Trapezoidation + Area Guards

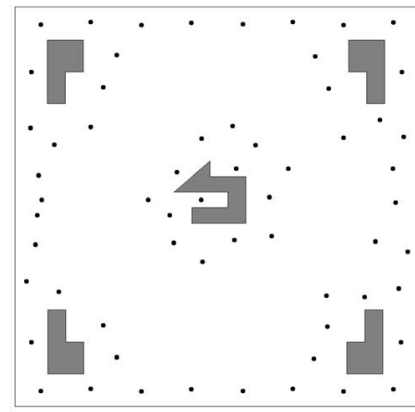

(c) Boundary Guards

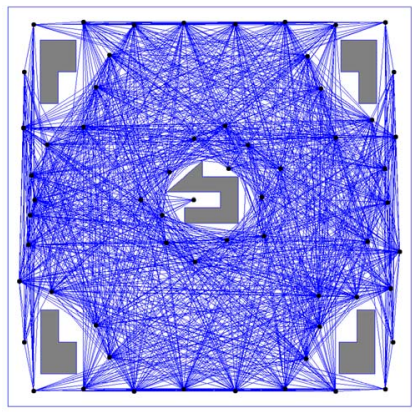

(d) Boundary Graph

Fig. 1: Sequential Stages of Building the Boundary Graph

posed: (1) Uninformed Boundary Coverage and (2) Informed Boundary Coverage.

Uninformed Boundary Coverage uses a heuristic algorithm for the Travelling Salesman Problem to patrol the boundaries. On the other hand, Informed Boundary Coverage is primarily based on an algorithm in which each robot autonomously and continuously learns the pattern of event occurrence on the boundaries over time, capturing the uncertainties in the target area. Based on the policy being learned to maximize the reward, each robot then plans in a distributed manner to select the best possible path at the time in the target area to visit the most promising parts of the boundary. Informed Boundary Coverage is an online learning algorithm.

The performance of the proposed approaches are evaluated on the basis of the total reward received by the team during a finite repeated boundary coverage mission. Moreover, the effects of robots' visual range and communication among the robots on the performance of the proposed algorithms are also investigated.

\section{BACKGROUND AND REVIEW}

Elmaliach et al. [8] addressed the problem of frequencybased patrolling (i.e. maximizing the minimum, maximum, or uniform point-visit frequency) of open polylines (e.g. as in open-ended fences), where the two endpoints of the polylines are not connected. They also investigated the velocity uncertainties and accumulating motion errors of the robots during the mission. Jensen et al. [9] extended Elmaliach et al.'s work on patrolling open polylines, with a focus on maintaining the patrol over the long-term. They accomplished this task by replacing the robots having power level below a threshold with some reserve robots. Marino et al. [10] proposed a decentralized multi-robot approach to patrol both open and closed polylines.

The patrolling problem, as studied in the above papers, is investigated in terms of optimizing point-visit frequency. Some other work considered the existence of adversary agents in the workspace. For instance, Agmon et al. [6], [7] studied patrolling a cyclic border, in which the robots' goal was to maximize their rewards by detecting an adversary agent, attempting to penetrate through a point on the boundary, unknown to the robots. The intruder needed some time interval of length $t$ to accomplish the intrusion. Jurek Czyzowicz et al. [11] addressed the same problem using a team of variablespeed robots.

As far as the authors are aware, there is no work using the framework studied in this paper. In our work, instead of patrolling a single open or closed polyline, the robots patrol the boundaries of a full environment and the structures inside it, and instead of optimizing frequency criteria, it is assumed that different parts of the boundary may have different priorities depending on the probability distribution of events occurrence on the boundaries. Finally, our robots aim to detect multiple events/intruders simultaneously, as opposed to single intruder scenarios studied in the previous work.

\section{ENVIRONMENT Modeling}

Uninformed Boundary Coverage and Informed Boundary Coverage both require that a roadmap is built within the target area, capturing the connectivity of the free space close to the boundaries. To this end, a graph-based representation called the Boundary Graph is constructed on the target area. The Boundary Graph enables the robots to move throughout the environment to monitor the boundaries of the area and the structures inside. Since the environment is known to the robots, each robot can independently build the Boundary Graph in the target area. In order to construct the Boundary Graph, a sufficient number of control points, called the boundary guards, are placed within the environment, considering the limited visual range of the robots.

\section{A. Locating Guards with Limited Visual Range}

In our problem definition, we presume the robots are equipped with panoramic cameras with a $360^{\circ}$ field of view. However, the cameras' visual range is limited. The proposed approach initially locates a set of area guards required to visually cover an entire area. The term guard is taken from the Art Gallery Problem [12]. These static area guards are control points that can jointly cover the whole environment while satisfying the limited visual range constraint of the robots. In other words, if we had as many robots as the number of guards, and each robot was stationed on a guard, the entire area would be covered visually by the robots.

To locate the guards, the algorithm decomposes the initial target area (Figure 1a), a 2D simple polygon with static structures, into a collection of convex polygons using a Trapezoidal Decomposition method, and then applies a post-processing 
approach to eliminate as many trapezoids as possible [13] (Figure 1b). The post-processing step is more effective in cluttered areas, and since the number of guards located by the algorithm is directly related to the number of trapezoids, fewer trapezoids will result in fewer guards.

At the next step, a divide-and-conquer method [14] is used to successively subdivide each of the resulting convex polygons (trapezoids) into smaller convex sub-polygons until each of them can be covered visually by one guard (Figure 1b).

Since, in the current problem, we are interested in monitoring only the boundaries, not all the computed area guards are necessary. So, each guard such that the visual area of a robot does not intersect the boundaries when it is located on that guard is removed from the set of area guards. Figure 1c illustrates the boundary guards $(B G)$ computed on the sample environment.

$$
B G=\left\{g_{1}, g_{2}, \ldots, g_{k}\right\} .
$$

\section{B. Boundary Graph}

Once the boundary guards are located in the target area, a graph called the Visibility Graph $(V G)$ is constructed on the guards (Figure 1d). In order to build the Visibility Graph, any pair of boundary guards which are mutually visible are connected by an edge. Two guards are mutually visible if the edge connecting them does not intersect any structures in the environment.

Visibility Graph is used to build the roadmap, because it provides the robots with more paths and more freedom of movement to traverse the environment, compared to other approaches such as Constrained Delaunay Triangulation or Voronoi Diagram.

\section{Boundary Segmentation}

The boundaries of the area and the structures are divided into identical length segments, each of which is small enough such that if an event occurs in a segment, the event is visible from any part of that segment. In other words, if a robot's visual range covers just part of the segment on the boundary, the robot is still capable of detecting all the events occurred in any part of the segment.

$$
\text { Segments }=\left\{\operatorname{seg}_{1}, \operatorname{seg}_{2}, \ldots, \operatorname{seg}_{n}\right\} .
$$

Definition 3. Visual Area of a Guard (VA): The visual area of a guard, $V A(\mathrm{~g})$, is the set of all the segments visible to the robot when it is located on the guard $g$.

$$
V A(g)=\left\{\operatorname{seg}_{i}, \operatorname{seg}_{j}, \ldots, \operatorname{seg}_{s}\right\} .
$$

Definition 4. Shared Segment: A shared segment is common to the visual area of two or more guards.

Definition 5. Segment Parent Guards (SPG): Parent guards of a shared segment are guards whose visual area contain that segment.

$$
S P G(\operatorname{seg})=\left\{g_{i}, g_{j}, \ldots, g_{p}\right\} .
$$

The notion of segment parent guards implies that an event occurred in a segment can be detected from more than one

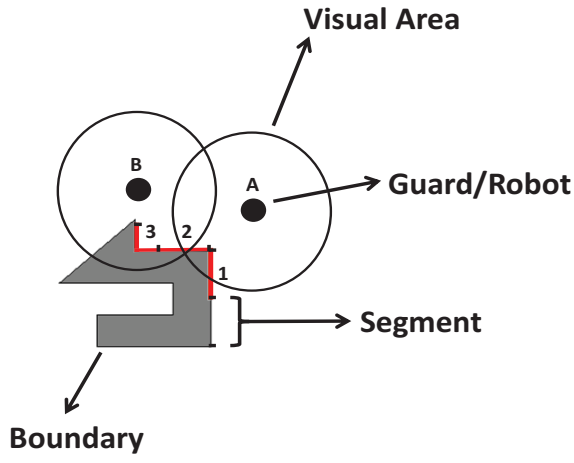

Fig. 2: Boundary Segmentation

guard, but once it is detected from a guard, the event is no longer visible from the other guards.

Assumption 1. Events are only detected when a robot is located on a guard.

In Figure 2, the visual area of guard $A$ covers segments 1 and 2 , and the visual area of guard $B$ covers segments 2 and 3 . Segment 2 is a shared segment between guards $A$ and $B$, and subsequently, guards $A$ and $B$ are the parent guards of segment 2. A robot located on guard $A$ can detect the events occurred in any part of segments 1 and 2, and a robot located on guard $B$ can detect the events occurred in any part of segments 2 and 3 .

\section{UNINFORMED BOUNDARY COVERAGE}

In Uninformed Boundary Coverage, a tour is constructed on the Boundary Graph using the Chained Lin-Kernighan algorithm.

Chained Lin-Kernighan [15], a modification of the LinKernighan algorithm [16], is generally considered to be one of the best heuristic methods for generating optimal or nearoptimal solutions for the Euclidean Traveling Salesman Problem. Given the distance between each pair of a finite number of nodes in a complete graph, the Travelling Salesman Problem is to find the shortest tour passing through all the nodes exactly once and returning to the starting node.

The input of the Chained Lin-Kernighan algorithm is the distance matrix of the Boundary Graph. The matrix consists of the shortest path distances between all pairs of guards in the Boundary Graph, and is consequently indicative of a complete graph, even though the Boundary Graph itself is not complete. Having built the shortest tour passing through all the guards of the Boundary graph, the robots are then distributed equidistantly along the tour and move repeatedly around it in the same direction.

\section{INFORMED BOUNDARY COVERAGE}

For Informed Boundary Coverage, the robots try to maximize the reward by detecting the maximum number of events, weighted by their importance, in minimum time. To this end, each robot independently learns the pattern of event occurrence on the boundaries over time and based on that, estimates the expected reward of visiting a state in the target area at each time step. Each robot then plans in a decentralized manner to select the best possible path to visit the most 
promising states at the time in the target area. The initial locations of the robots are chosen randomly in the target area.

The Multi-Robot Repeated Boundary Coverage problem is formulated for each robot as a tuple $(B G, A, S T, S T R)$ where:

- $B G$ is the set of states or boundary guards, representing the position of the robot in the target area.

- $A$ is the set of actions available for a robot in each state. An action is defined as moving from one guard to any other guard in the Boundary Graph. At the beginning, each robot calculates the shortest path between each pair of guards in the Boundary Graph, using the FloydWarshall algorithm, hence the robots will not need to repeatedly compute the shortest paths in the graph during the planning stage of the coverage mission.

- $S T$ is the state transition function which is deterministic, such that it guarantees reaching the target state chosen by the robot from the current state, when the action is performed.

- $S T R$ is the state reward at a particular time, which is equal to the sum of the discounted importance of the events at the state (i.e. guard):

$$
\operatorname{STR}(g, t)=\sum_{s e g \in V A} \sum_{(g)} \sum_{E_{i} \in E} \text { weight }\left(E_{i}\right) \times E_{i} \gamma^{t-s t\left(e_{i}\right)},
$$

where $t-s t(e)$ is the time interval between starting event $e$ and the time $t$. Once a robot arrives at a guard $g$, it can detect all the events occurred within the $V A(g)$, the visual area of the guard $g$. It is assumed that the reward a robot receives for an event depends on how early the event is detected. At each time step after the event occurrence, the detection reward of the event is multiplied by a discount factor of $\gamma=0.95$.

Definition 6. Time of Last Visit (TLV): Each robot separately keeps track of the times of the last visit to the guards. If $B G=\left\{g_{1}, g_{2}, \ldots, g_{k}\right\}$ is the set of boundary guards, then for each guard $g \in B G, T L V(g)$ represents the last time the guard $g$ was visited by the robot or by any other robot who managed to communicate its visit to the guard to that robot. Therefore, the times of the last visit to the guards are not globally shared by the robot team, rather each robot, at each time step, may have a different beliefs from the rest of the robot team of the times of the last visit to the guards.

Based on this, we can calculate the time of the last visit to each segment of the boundary:

$$
T L V(\operatorname{seg})=\max \{T L V(g) \mid g \in S P G(\operatorname{seg})\} .
$$

Intuitively, the time of the last visit to a segment is the most recent visit of the robot to one of the segment's parent guards.

Definition 7. Policy: A policy $\pi(r): B G \rightarrow A$ at each state determines which action should be performed next by robot $r$.

Note that the learning procedure described below is performed by each robot independently of the rest of the team.

\section{A. Learning}

If the robot had complete knowledge of the starting time of the events in each state, it would be able to calculate the STR to find a policy, maximizing the total reward of the boundary coverage mission, but since this information is not available to the robot, it estimates the STR as the sum of the Expected Segment Reward (ESR) of the segments comprising a state:

$$
\operatorname{STR}(g, t) \simeq \sum_{s e g \in V A(g)}(\operatorname{ESR}(\operatorname{seg}, t)) .
$$

Expected Segment Reward (ESR) is defined to represent the expected reward of a segment, seg, at the time $t$ :

$$
\begin{aligned}
& \operatorname{ESR}(\operatorname{seg}, t)=\sum_{E_{i} \in E} \sum_{e_{i} \in E_{i}}\left(1+\gamma^{1}+\gamma^{2}+\ldots+\gamma^{t-T L V(s e g)}\right) \times \\
& \operatorname{PSE}\left(E_{i}, \operatorname{seg}\right) \times \operatorname{weight}\left(E_{i}\right),
\end{aligned}
$$

where $\gamma$ is the reward discount factor. We assume that for every time step after an event occurs without being detected, the event detection reward is discounted by $\gamma$. Furthermore, the Probability of Segment Event (PSE) is defined for each event type $E_{i} \in E$ and each segment, seg, to indicate the probability of events of type $E_{i}$ occur within the segment at each time step.

In equation (8), $\sum_{E_{i} \in E} \sum_{e_{i} \in E_{i}} \operatorname{PSE}\left(E_{i}, \operatorname{seg}\right) \times \operatorname{weight}\left(E_{i}\right)$ is the Segment Reward Accumulation Rate of the events in the segment, seg, and is represented by $\operatorname{SRAR}(\mathrm{seg})$. If a robot knows the SRAR of the events in a segment, it can calculate the segment's ESR for any arbitrary time $t$.

1) Estimating the SRAR of the Segments: In the initialization step, the robot assumes the SRAR of all the segments is 1 . A learning procedure for estimating the $S R A R$ of the segments gradually updates their initial value. When the robot arrives at a guard $g$, it can detect whether or not an event has occurred at the segments belonging to $V A(g)$. The $S R A R$ of the guard's segments is then updated using the following equation:

$$
\begin{aligned}
& \forall \operatorname{seg} \in \operatorname{VA}(g), \operatorname{SRAR}(\mathrm{seg})= \\
& (1-\alpha) \times \operatorname{SRAR}(\mathrm{seg})+\alpha \times \frac{\sum_{E_{i} \in E} \sum_{e_{i} \in E_{i}}\left(\text { weight }\left(E_{i}\right)\right)}{t(g)-T L V(\operatorname{seg})},
\end{aligned}
$$

where $\alpha$ is the learning rate set to 0.9 and $t(g)$ is the time of the visit to the guard $g$. This equation gives more weight to the new information than the old information. The robot performs the updating process for all the event types and all the segments of the guards.

In summary, the SRAR of a segment is updated once a robot visits one of its parent guards. As already mentioned, the $S R A R$ represents the reward accumulation rate in a segment. Now we can use the $S R A R$ to estimate the ESR of the segments using the following procedure.

2) Estimating the ESR of the Segments: At the beginning of the mission, the robot initializes the ESR of all the segments to zero. Then, at each time step, if the robot has yet to arrive at a guard, the ESR of all the segments of the boundary is updated using the following equation:

$$
\begin{aligned}
& \forall \text { seg } \in \text { Segments, } \\
& \operatorname{ESR}(\operatorname{seg}, t)=\gamma \times \operatorname{ESR}(\operatorname{seg}, t-1)+\operatorname{SRAR}(\operatorname{seg}) .
\end{aligned}
$$

If the robot arrives at a guard $g$, it detects all the events that have occurred in its segments and communicates the guard ID 


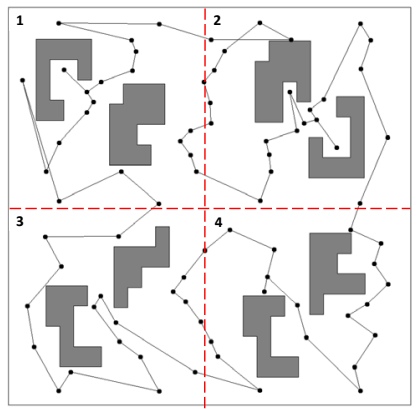

(a) Map 1

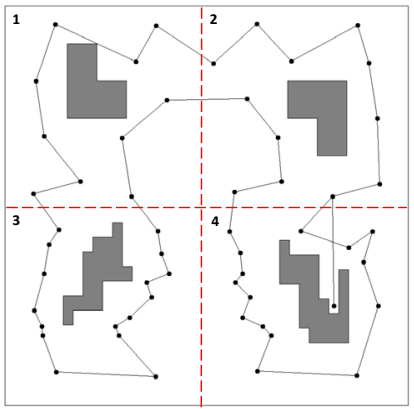

(b) Map 2

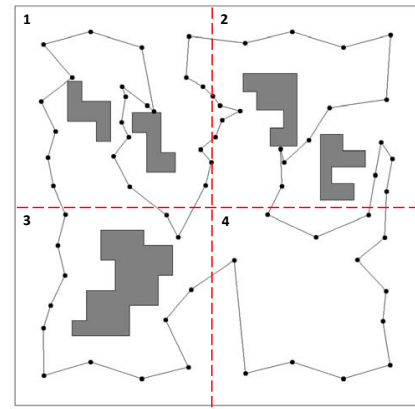

(c) Map 3

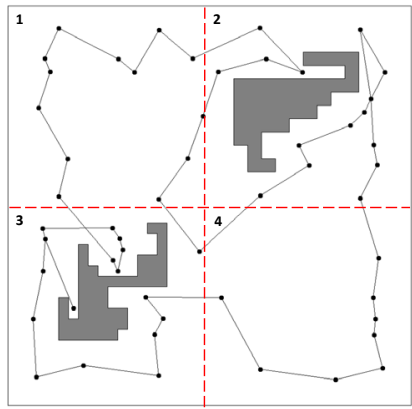

(d) Map 4

Fig. 3: Maps Used in the Experiments

to the robots located within its communication range. Since all the events occurred in the segments of $g$ have been detected, the expected reward of the segments at the time of the visit to the guard, $t(g)$, becomes zero. Consequently, for the robot and all the communicated robots:

$$
\forall \operatorname{seg} \in V A(g), \operatorname{ESR}(\operatorname{seg}, t(g))=0 .
$$

Note that each robot has its own knowledge of the ESR of the segments, so the other robots (except the ones who received the communication) may still assume that there are some undetected events in the segments of $g$ at the time, and subsequently their ESRs of the segments of $g$ are not zero.

In the Experiments and Results section (Section VI), we will show that how communication among the robots influences the performance of the algorithm and compare it with the case that robots do not communicate or share any knowledge of the environment during the operation.

The updating process of the $S R A R$ and the ESR of the segments continues during the boundary coverage operation.

\section{B. Planning}

Once a robot arrives at a guard and detects all the events which may have occurred in the segments of the guard, the robot selects the next action to perform. As already mentioned, an action in the boundary coverage operation is defined as moving from one guard to another guard in the Boundary Graph. At each state, the robot considers all the precomputed shortest paths to all the other guards it can move to. For each path, $\operatorname{path}\left(g_{c}, g_{d}\right)$, where $g_{c}$ is the current guard and $g_{d}$ is the destination guard, Path Reward $(P R)$ is defined as the reward the robot receives when moving from the guard $g_{c}$ to the guard $g_{d}$. The path from $g_{c}$ to $g_{d}$ includes zero or more intermediate guards and can be represented as:

$$
\operatorname{path}\left(g_{c}, g_{d}\right)=\left[g_{c}, g_{i}, g_{j}, \ldots, g_{r}, g_{d}\right] .
$$

Given the speed of the robot, the arrival time at each of the guards on the path can be estimated. Hence, the robot can have an estimate of the $\operatorname{ESR}(\mathrm{seg}, t(\mathrm{~g}))$ for each segment of the guard $g \in \operatorname{path}\left(g_{c}, g_{d}\right)$, in which $t(g)$ is the arrival time to the guard $g$. For such a path, the $P R$ is calculated as below:

$$
P R\left(\operatorname{path}\left(g_{c}, g_{d}\right)\right)=\sum_{g \in \operatorname{path}\left(g_{c}, g_{d}\right)} \sum_{\operatorname{seg} \in V A(g)} E S R(\operatorname{seg}, t(g)) .
$$

When calculating the $P R$, the robot should take into account the segments shared by some parent guards as well, namely the robot in its calculations initializes the shared segment's ESR to zero when it is going to visit one of its parent guards along the path.

Next, for each path, the Average Path Reward (APR) is calculated using the following equation:

$$
\operatorname{APR}\left(\operatorname{path}\left(g_{c}, g_{d}\right)\right)=\frac{P R\left(\operatorname{path}\left(g_{c}, g_{d}\right)\right)}{t\left(g_{d}\right)-T L V\left(g_{c}\right)},
$$

where $g_{c}$ is the current guard, $T L V\left(g_{c}\right)$ is the current time or the time of the last visit to the guard $g_{c}, g_{d}$ is the destination guard, and $t\left(g_{d}\right)$ is the arrival time to the guard $g_{d}$. The robot will select a path with the maximum Average Path Reward to traverse next.

\section{EXPERIMENTS AND RESULTS}

We wish to compare Uninformed Boundary Coverage $(U B C)$ with the two variants of Informed Boundary Coverage $(I B C)$ (i.e. non-communicating and communicating robot teams) in terms of the total reward being received by the team for detecting the events in a finite simulation time. We have developed a simulator to test the algorithms in different scenarios. The simulator can support different numbers of robots in the target area, different visual ranges for the robots, and varying degrees of clutter in the environment.

The experiments are conducted using 10 robots on the sample environments of Figure 3. The size of the environments is $10 \mathrm{~m} \times 10 \mathrm{~m}$, and the boundaries are divided into segments of length $1 \mathrm{~m}$. We divide the environment maps into 4 disjoint sub-regions. In maps 1 and 2, there are structures in all subregions of the environment. In map 3, there are structures in 3 of the sub-regions, and in map 4, there are structures in 2 of the sub-regions. Three different visual ranges are also considered for the robots: $0.25 \mathrm{~m}$ (low), $0.75 \mathrm{~m}$ (medium), $1.50 \mathrm{~m}$ (high). The communication range of the robots is $1.5 \mathrm{~m}$. Figure 3 also shows, as a sample, the cyclic tours built on the maps using the Uninformed Boundary Coverage algorithm, assuming that the robots' visual range is $0.75 \mathrm{~m}$.

4 experiments were designed, in each, the pattern of event occurrence varies in the sub-regions. Figures 4, 5, 6 and 7 show the total reward received by the team (10 robots) in each experiment on map 1 under different visual ranges. The other maps show a similar trend to that for map 1. 


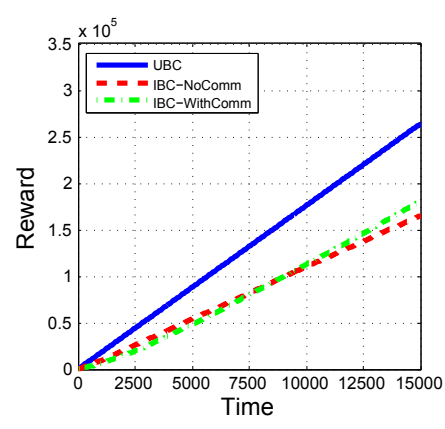

(a) Visual Range $=0.25 \mathrm{~m}$

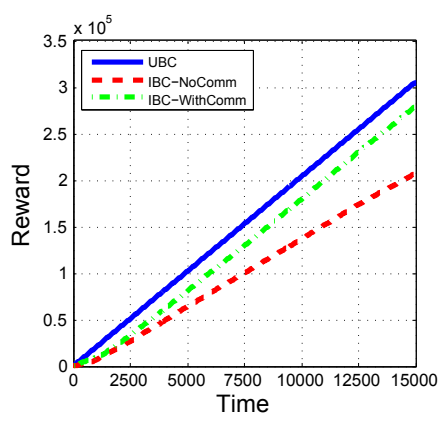

(b) Visual Range $=0.75 \mathrm{~m}$

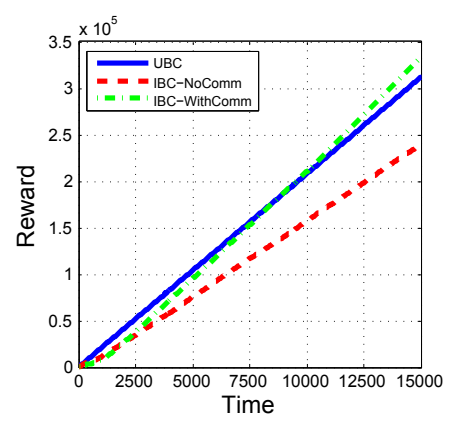

(c) Visual Range $=1.50 \mathrm{~m}$

Fig. 4: Experiment 1: Uniform Event Occurrence

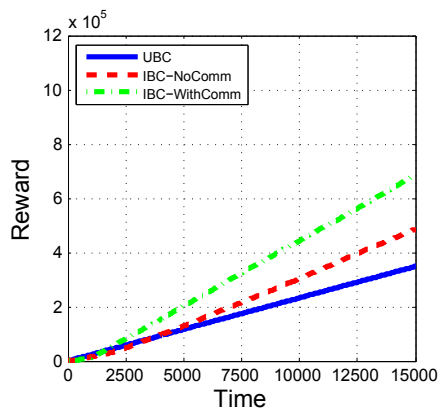

(a) Visual Range $=0.25 \mathrm{~m}$

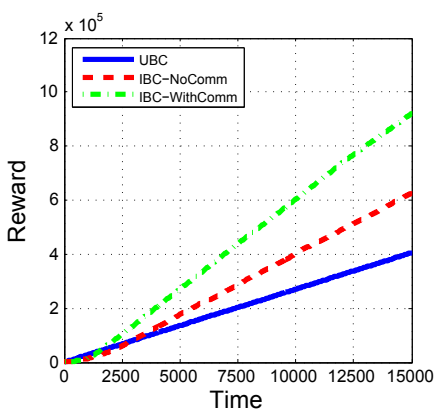

(b) Visual Range $=0.75 \mathrm{~m}$

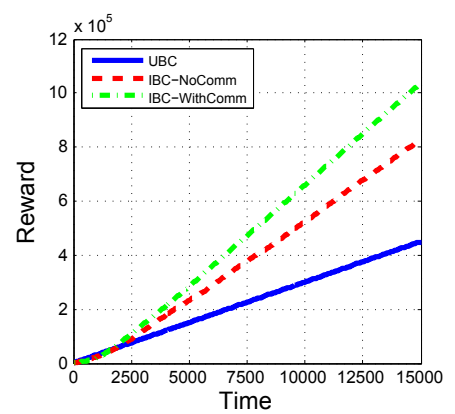

(c) Visual Range $=1.50 \mathrm{~m}$

Fig. 5: Experiment 2: Non-uniform Event Occurrence

\section{A. Experiment 1: Uniform Event Occurrence}

In this experiment, the events occur in all the segments of the boundaries, and in all the sub-regions of the environment uniformly with an equal probability of 0.5 , meaning that at each cycle there is a 0.5 chance that an event occurs in a segment. Each event has a weight of 1 .

As shown in Figure 4, Uninformed Boundary Coverage collects more rewards than Informed Boundary Coverage in low and medium visual ranges, however as the visual range increases, the difference between the two algorithms declines, and even in high visual range of $1.50 \mathrm{~m}$, the communicating version of Informed Boundary Coverage outperforms Uninformed Boundary Coverage. Also, communicating robots collect more rewards than non-communicating robots in Informed Boundary Coverage. Finally, increasing the robots' visual range leads to all the algorithms collect more rewards after a 15000 cycle run of the simulation.

\section{B. Experiment 2: Non-uniform Event Occurrence}

In this experiment, no events occur on the boundaries of sub-region 1. Within sub-region 2 the events occur with a probability of 0.1 in each segment of the boundaries. Within sub-region 3 , the events occur with a probability of 0.9 , and within sub-region 4 , the events occur according to a Poisson distribution with a mean of $\lambda=4$. This implies that more than one event can occur in each segment at every cycle. Each event occurring in sub-regions 2 and 3 is weighted 1 , and 0.5 in sub-region 4.
As shown in Figure 5, Informed Boundary Coverage outperforms Uninformed Boundary Coverage regardless of the robots' visual range, and as the visual range increases, the difference between the two algorithms grows. Also, communicating robots collect more rewards than non-communicating robots in Informed Boundary Coverage. Furthermore, increasing the robots' visual range leads to all the algorithms collect more rewards after a 15000 cycle run of the simulation.

\section{Experiment 3: All Events Occur in One Sub-region}

In this experiment, in sub-region 1 , the events occur with a probability of 0.9 in the segments of the boundary. No events occur in sub-regions 2, 3 and 4. Each event is weighted 1.

As shown in Figure 6, Informed Boundary Coverage outperforms Uninformed Boundary Coverage regardless of the robots' visual range, and as the visual range increases, the difference between the two algorithms grows. Also, communicating robots collect more rewards than non-communicating robots in Informed Boundary Coverage. Furthermore, increasing the robots' visual range leads to all the algorithms collect more rewards after a 15000 cycle run of the simulation.

\section{Experiment 4: Dynamic Event Occurrence}

In this experiment, the pattern of event occurrence changes at some times unknown to the robots during the simulation run. We presume that during the first 8000 cycles, the events occur in the environment according to the pattern discussed in Experiment 1 (Uniform Event Occurrence), between cycles 


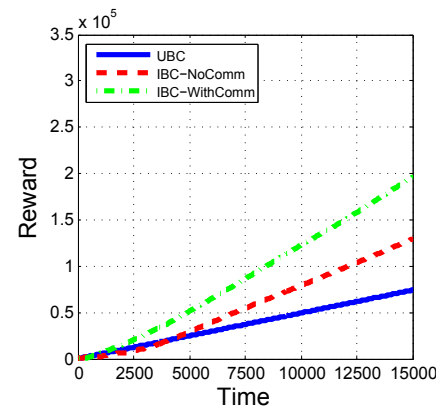

(a) Visual Range $=0.25 \mathrm{~m}$

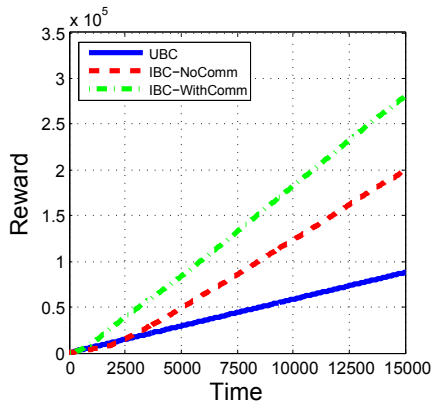

(b) Visual Range $=0.75 \mathrm{~m}$

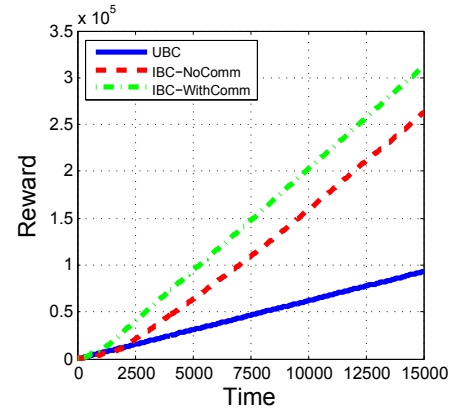

(c) Visual Range $=1.50 \mathrm{~m}$

Fig. 6: Experiment 3: All Events Occur in One Sub-region

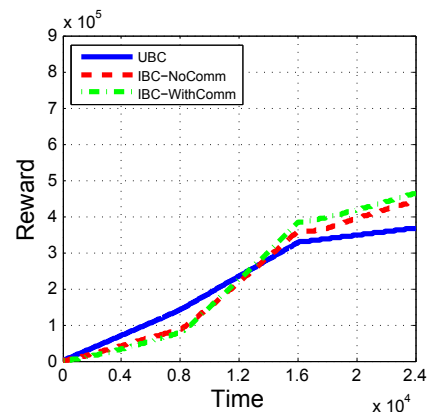

(a) Visual Range $=0.25 \mathrm{~m}$

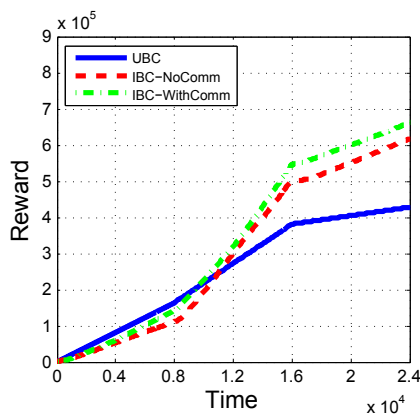

(b) Visual Range $=0.75 \mathrm{~m}$

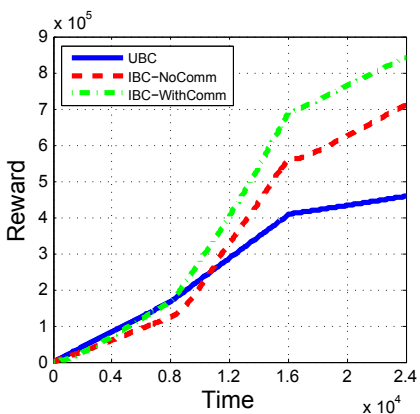

(c) Visual Range $=1.50 \mathrm{~m}$

Fig. 7: Experiment 4: Dynamic Event Occurrence

$8000-16000$, the events occur according to the pattern discussed in Experiment 2 (Non-uniform Event Occurrence), and between cycles $16000-24000$, the events occur according to the pattern mentioned in Experiment 3 (All Events Occur in One Sub-region).

As shown in Figure 7, Informed Boundary Coverage outperforms Uninformed Boundary Coverage regardless of the robots' visual range, and as the visual range increases, the difference between the two algorithms grows. Also, communicating robots collect more rewards than non-communicating robots in Informed Boundary Coverage. Furthermore, increasing the robots' visual range leads to all the algorithms collect more rewards after a 24000 cycle run of the simulation. In this experiment, the robots adapted themselves to the changes in the pattern of event occurrence on the boundaries, and updated their policies based on these changes.

\section{E. Discussion}

Tables $8 \mathrm{a}, 8 \mathrm{~b}$, and $8 \mathrm{c}$ show the total reward received by the team using Uninformed Boundary Coverage $(U B C)$ and the two variants (i.e. communicating and non-communicating robots) of Informed Boundary Coverage (IBC) on the 4 maps of Figure 3 under visual ranges of $0.25 \mathrm{~m}, 0.75 \mathrm{~m}$, and $1.50 \mathrm{~m}$. The results on maps 2,3 and 4 are consistent with the results discussed for map 1. As shown in the tables, Uninformed Boundary Coverage collected more rewards on all the maps in low and medium visual ranges, when the events occur uniformly along the boundaries (Experiment 1). How- ever, Informed Boundary Coverage outperforms Uninformed Boundary Coverage on all the maps when the events occur uniformly and the robots' visual range is high, or when the events occur non-uniformly, on just parts of the boundaries, or when the pattern of event occurrence changes over time regardless of the robots' visual range.

\section{CONClusions AND Future WORK}

We have addressed the problem of repeated coverage by a team of robots of the boundaries of a target area and the structures inside it. The robots have limited circular visual and communication range. Events may occur on any parts of the boundaries and may have different importance weights. The robots are not a priori aware of the probability distribution of occurrence of events on the boundaries. As a result, each robot autonomously and continuously learns the pattern of event occurrence on the boundaries over time, capturing the uncertainties in the target area, and then plans in a decentralized manner to select the best path at that time to visit the most promising parts of the boundary. The performance of the learning algorithm was compared with a heuristic algorithm for the Travelling Salesman Problem, on the basis of the total reward collected by the team during a finite repeated boundary coverage mission. Moreover, the effects of the robots' visual range and communication among the robots on the performance of the proposed algorithms were also investigated.

For future work, we plan to address issues related to 


\begin{tabular}{|c|c|c|c|c|c|c|c|c|c|c|c|c|c|}
\hline & \multirow[b]{2}{*}{$\begin{array}{c}\text { \# of } \\
\text { Robots }\end{array}$} & \multicolumn{3}{|c|}{ Experiment 1} & \multicolumn{3}{|c|}{ Experiment 2} & \multicolumn{3}{|c|}{ Experiment 3} & \multicolumn{3}{|c|}{ Experiment 4} \\
\hline & & UBC & $\begin{array}{c}\text { IBC- } \\
\text { NoComm }\end{array}$ & $\begin{array}{c}\text { IBC- } \\
\text { WithComm }\end{array}$ & UBC & $\begin{array}{c}\text { IBC- } \\
\text { NoComm }\end{array}$ & $\begin{array}{c}\text { IBC- } \\
\text { WithComm }\end{array}$ & UBC & $\begin{array}{c}\text { IBC- } \\
\text { NoComm }\end{array}$ & $\begin{array}{c}\text { IBC- } \\
\text { WithComm }\end{array}$ & UBC & $\begin{array}{c}\text { IBC- } \\
\text { NoComm }\end{array}$ & $\begin{array}{c}\text { IBC- } \\
\text { WithComm }\end{array}$ \\
\hline Map \#1 & 10 & 263490 & 164490 & 180080 & 348220 & 485240 & 685400 & 74086 & 129090 & 196070 & 367460 & 441640 & 463710 \\
\hline Map \#2 & 10 & 241310 & 143630 & 156180 & 398280 & 507880 & 726660 & 49793 & 95509 & 123820 & 368950 & 433640 & 462910 \\
\hline Map \#3 & 10 & 256470 & 166920 & 170740 & 252340 & 363090 & 449060 & 79852 & 140790 & 211140 & 315210 & 371540 & 407870 \\
\hline Map \#4 & 10 & 152500 & 162100 & 278690 & 362380 & 502500 & 36539 & 95525 & 99411 & 295140 & 345730 & 385970 & 152500 \\
\hline
\end{tabular}

(a) Visual Range $=0.25 \mathrm{~m}$

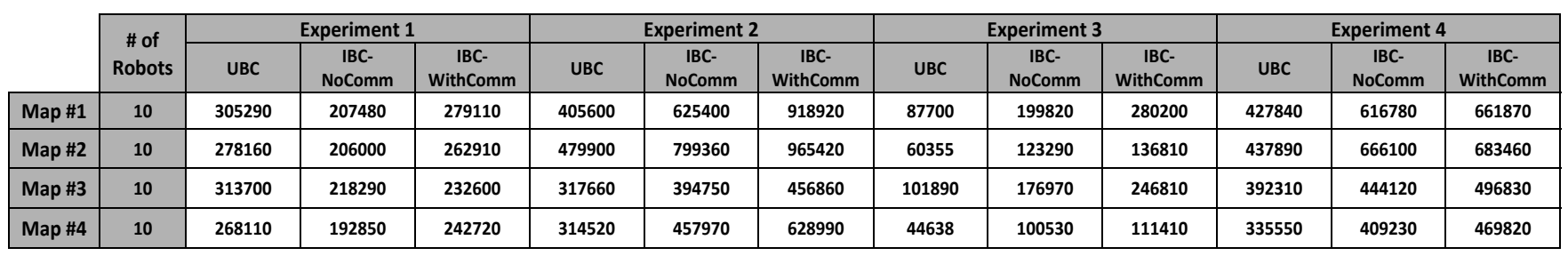

(b) Visual Range $=0.75 \mathrm{~m}$

\begin{tabular}{|c|c|c|c|c|c|c|c|c|c|c|c|c|c|}
\hline & \multirow[b]{2}{*}{$\begin{array}{l}\text { \# of } \\
\text { Robots }\end{array}$} & \multicolumn{3}{|c|}{ Experiment 1} & \multicolumn{3}{|c|}{ Experiment 2} & \multicolumn{3}{|c|}{ Experiment 3} & \multicolumn{3}{|c|}{ Experiment 4} \\
\hline & & UBC & $\begin{array}{c}\text { IBC- } \\
\text { NoComm }\end{array}$ & $\begin{array}{c}\text { IBC- } \\
\text { WithComm } \\
\end{array}$ & UBC & $\begin{array}{c}\text { IBC- } \\
\text { NoComm }\end{array}$ & $\begin{array}{c}\text { IBC- } \\
\text { WithComm } \\
\end{array}$ & UBC & $\begin{array}{c}\text { IBC- } \\
\text { NoComm }\end{array}$ & $\begin{array}{c}\text { IBC- } \\
\text { WithComm } \\
\end{array}$ & UBC & $\begin{array}{c}\text { IBC- } \\
\text { NoComm }\end{array}$ & $\begin{array}{c}\text { IBC- } \\
\text { WithComm }\end{array}$ \\
\hline Map \#1 & 10 & 311260 & 239220 & 332450 & 449400 & 826490 & 1036000 & 92864 & 262190 & 311380 & 457840 & 709760 & 842120 \\
\hline Map \#2 & 10 & 285810 & 232410 & 289810 & 520030 & 1012100 & 1403600 & 64843 & 164620 & 172460 & 465580 & 772580 & 924670 \\
\hline Map \#3 & 10 & 377130 & 297420 & 385490 & 412210 & 604820 & 664600 & 130720 & 283410 & 328960 & 490230 & 641130 & 694760 \\
\hline Map \#4 & 10 & 259020 & 217570 & 306490 & 317470 & 586950 & 729790 & 45030 & 144560 & 149380 & 331900 & 519930 & 621320 \\
\hline
\end{tabular}

(c) Visual Range $=1.50 \mathrm{~m}$

Fig. 8: Total Reward Collected by the Team on Different Maps Based on Different Experiments and Various Visual Ranges

noisy sensors of the robots, action uncertainty, and unknown structures. In the case of noisy sensors, the accuracy of the information achieved by a robot could vary with the distance of the boundary from the robot. Some events might also require multiple robots to form a coalition in order to be handled. Another future direction is the case that the robot team would have the ability to change its behavior over time in response to a changing environment with dynamic structures, either to improve performance or to prevent unnecessary degradation in performance.

\section{REFERENCES}

[1] P. Fazli and A. Mackworth, "Multi-robot repeated boundary coverage under uncertainty," in Proceedings of the IEEE International Conference on Robotics and Biomimetics, ROBIO, 2012.

[2] P. Fazli, A. Davoodi, P. Pasquier, and A. K. Mackworth, "Complete and robust cooperative robot area coverage with limited range," in Proceedings of the IEEE/RSJ International Conference on Intelligent Robots and Systems, IROS, 2010, pp. 5577-5582.

[3] P. Fazli, A. Davoodi, and A. Mackworth, "Multi-robot repeated area coverage: Performance optimization under various visual ranges," in Proceedings of the Ninth Conference on Computer and Robot Vision, CRV, 2012, pp. $298-305$.

[4] M. Ahmadi and P. Stone, "A multi-robot system for continuous area sweeping tasks," in Proceedings of the IEEE International Conference on Robotics and Automation, ICRA, 2006, pp. 1724-1729.

[5] P. Amstutz, N. Correll, and A. Martinoli, "Distributed boundary coverage with a team of networked miniature robots using a robust market-based algorithm," Annals Mathematics Artificial Intelligence, vol. 52, no. 2-4, pp. 307-333, 2008.

[6] N. Agmon, S. Kraus, and G. Kaminka, "Multi-robot perimeter patrol in adversarial settings," in Proceedings of the IEEE International Conference on Robotics and Automation, ICRA, 2008, pp. 2339-2345.
[7] N. Agmon, S. Kraus, G. A. Kaminka, and V. Sadov, "Adversarial uncertainty in multi-robot patrol," in Proceedings of the International Joint Conference on Artificial Intelligence, IJCAI, 2009, pp. 1811-1817.

[8] Y. Elmaliach, A. Shiloni, and G. A. Kaminka, "A realistic model of frequency-based multi-robot polyline patrolling," in Proceedings of the 7th International Joint Conference on Autonomous Agents and Multiagent Systems, AAMAS, 2008, pp. 63-70.

[9] E. Jensen, M. Franklin, S. Lahr, and M. Gini, "Sustainable multi-robot patrol of an open polyline," in Proceedings of the IEEE International Conference on Robotics and Automation, ICRA, 2011, pp. $4792-4797$.

[10] A. Marino, L. Parker, G. Antonelli, and F. Caccavale, "Behavioral control for multi-robot perimeter patrol: a finite state automata approach," in Proceedings of the IEEE International Conference on Robotics and Automation, ICRA, 2009, pp. 3350-3355.

[11] J. Czyzowicz, L. Gasieniec, A. Kosowski, and E. Kranakis, "Boundary patrolling by mobile agents with distinct maximal speeds," in Proceedings of the 19th European conference on Algorithms, ESA, 2011, pp. 701-712.

[12] J. O'Rourke, Art gallery theorems and algorithms. New York, NY, USA: Oxford University Press, Inc., 1987.

[13] B. Zalik and G. J. Clapworthy, "A universal trapezoidation algorithm for planar polygons," Computers \& Graphics, vol. 23, no. 3, pp. 353 363, 1999.

[14] G. D. Kazazakis and A. A. Argyros, "Fast positioning of limited visibility guards for inspection of 2D workspaces," in Proceedings of the IEEE/RSJ International Conference on Intelligent Robots and Systems, IROS, 2002, pp. 2843-2848.

[15] D. Applegate, W. Cook, and A. Rohe, "Chained Lin-Kernighan for large traveling salesman problems," INFORMS Journal on Computing, vol. 15, pp. 82-92, 2003.

[16] S. Lin and B. Kernighan, "An effective heuristic algorithm for the traveling-salesman problem," Operations Research, vol. 21, no. 2, pp. 498-516, 1973. 